\title{
Téoros
}

Revue de recherche en tourisme

\section{Incursion du côté des visiteurs dans le réseau des musées au Québec}

\section{Yves Bergeron et Suzanne Dumas}

Volume 27, numéro 3, automne 2008

Nouveaux Musées, Nouveaux Tourismes

URI : https://id.erudit.org/iderudit/1070781ar

DOI : https://doi.org/10.7202/1070781ar

Aller au sommaire du numéro

Éditeur(s)

Université du Québec à Montréal

ISSN

0712-8657 (imprimé)

1923-2705 (numérique)

Découvrir la revue

Citer cet article

Bergeron, Y. \& Dumas, S. (2008). Incursion du côté des visiteurs dans le réseau des musées au Québec. Téoros, 27(3), 29-34. https://doi.org/10.7202/1070781ar d'utilisation que vous pouvez consulter en ligne.

https://apropos.erudit.org/fr/usagers/politique-dutilisation/ 


\section{Incursion du côté des visiteurs dans le réseau des musées au Québec}

\section{Yves Bergeron et Suzanne Dumas}

\section{Les musées : un univers en mutation}

Au cours de la décennie 1990, l'univers des musées s'est profondément transformé. Depuis l'apparition en Europe des musées publics à la fin du XVIII e siècle, les musées s'étaient surtout préoccupés du développement de leurs collections permanentes, de sorte que la visite des musées était d'abord et avant tout centrée sur les expositions permanentes et la présentation des collections. Le musée était considéré comme un lieu de conservation de trésors et de chefsd'œuvre. Cette dynamique s'est peu à peu transformée dans un premier temps avec l'émergence des principes de la "nouvelle muséologie» au début des années 1960. Contrairement à l'Europe où le concept d'écomusée développé par George-Henri Rivière trouvait des échos favorables, les musées nord-américains empruntaient alors la voie proposée par Freeman Tilden qui préconise l'interprétation plutôt que la contemplation. Freeman Tilden (1957) propose cinq principes fondamentaux qui vont contribuer à transformer les musées : 1) se demander pourquoi les visiteurs viennent et ce qu'ils attendent du site ou du musée; 2) offrir un tout plutôt qu'une partie ; 3) ancrer une révélation dans la personnalité des visiteurs; 4) couler la matière brute dans des formes artistiques; et 5) se garder de tout excès. C'est ainsi qu'on assiste à un foisonnement des programmes éducatifs dans les musées d'histoire et les centres d'interprétation, de sorte que la nouvelle muséologie prend dès lors son envol aux États-Unis et au Canada. Les musées nord-américains se renouvellent et acquièrent de nouveaux publics. Conséquemment, on voit se multiplier au Québec au milieu des années 1980, comme ailleurs dans le monde, de plus en plus d'expositions temporaires, dans le but de maintenir l'intérêt des visiteurs. Les musées s'engagent d'ailleurs dans le remplacement des salles d'expositions permanentes par des salles d'expositions temporaires. Peu de temps après le début de ce mouvement, les musées nord-américains sont reconnus pour l'attention toute particulière qu'ils portent aux visiteurs. C'est dans cet esprit que Marshall McLuhan présente au musée de la ville de New York en 1967 une conférence sur les méthodes, les moyens et les valeurs de la communication avec le public, à l'occasion d'un colloque ayant pour thème « Museum Communication with the Viewing Public »'. Cette intervention s'inscrit dans l'esprit qui règne lors de l'Exposition universelle de Montréal en 1967. Cette sensibilité aux besoins et aux attentes des publics est d'ailleurs devenue une des caractéristiques fondamentale de la muséologie nord-américaine.

Si la grande majorité des musées québécois ont adopté les préceptes de la nouvelle muséologie, l'essentiel des principes prend forme dans le cadre de la création du Musée de la civilisation du Québec qui ouvre ses portes en 1988. Le musée intègre alors les principes de la nouvelle muséologie en se définissant comme un musée de société. Le directeur général du musée, Roland Arpin, a longuement écrit sur ce concept de muséologie de société (1992 et 1997). Peu de temps après son ouverture au public, le Musée de la civilisation devient un modèle qui inspire la création de nouveaux musées à l'échelle internationale. Mentionnons simplement le musée d'Orsay et le musée du quai Branly à Paris, le Musée des Confluences à Lyon, le Musée des Civilisations de l'Europe et de la Méditerranée à Marseille, le Musée d'histoire de l'Europe à Bruxelles et, plus récemment, le Musée national de la civilisation égyptienne qui ouvrira ses portes au Caire en 2009.

Les valeurs de la nouvelle muséologie se traduisent notamment dans les rapports que les musées entretiennent avec les publics. Ce mouvement culmine dans les musées nord-américains avec l'apparition de grandes expositions de type blockbuster ou "exposition vedette". On n'a qu'à penser à certaines expositions présentées par le Musée des beaux-arts de Montréal depuis le milieu des années 1980. Rappelons au passage les expositions suivantes : Le musée imaginaire de Tintin (1980), Les années 20 : l'âge des métropoles (1991), Catherine la Grande : un art pour l'Empire, Chefs-d'œuvre du Musée de l'Ermitage de Saint-Pétersbourg (2006) ou, plus récemment, II était une fois Walt Disney. Aux sources de l'art des studios Disney (2007). Mais le succès le plus marquant des expositions vedettes est sans aucun doute Rodin à Québec, exposition présentée par le Musée des beaux-arts du Québec, qui a fracassé tous les records de fréquentation en accueillant plus de 500000 visiteurs en quelques semaines. Ce phénomène, combiné à la multiplication des expositions thématiques, a eu pour effet de faire grimper en flèche la fréquentation des musées qui est passée de 31,2 \% à 39,1 \% de 1979 à 1999 (Garon et Santerre, 2004 : 56-57) Les directeurs ainsi que les conseils d'administration des grands musées ont compris que la multiplication des "expositions vedettes" a pour effet d'augmenter la fréquentation, qui se répercute directement sur les revenus de 
billetterie tout en augmentant l'aide financière en provenance du secteur privé. Ces nouvelles sources de financement dites "privées» se composent principalement de dons, de commandites et d'échanges de services. L'enquête menée par l'Observatoire de la culture et des communications du Québec (OCCQ) sur l'univers des institutions muséales révèle d'ailleurs que ce sont principalement les musées d'art qui bénéficient de telles retombées. Ce phénomène s'explique en raison du caractère prestigieux des œuvres et des expositions proposées par les musées d'art qui trouvent, plus facilement que les musées d'histoire ou les musées de sciences, des entreprises prêtes à s'associer à des projets prestigieux. On constate que le financement privé représente $23,5 \%$ du budget global des musées d'art alors qu'il atteint à peine 9,7\% dans le cas des musées d'histoire, d'ethnologie et d'archéologie et 2,9\% dans celui des musées de sciences (OCCQ, 2007). En somme, il devient clair pour les gestionnaires des musées que les «expositions vedettes» permettent de générer des revenus non négligeables, alors que les gouvernements incitent de plus en plus les musées à augmenter leur fréquentation et à diversifier leurs sources de financement (MCCQ, 2000). Cette nouvelle orientation a d'ailleurs permis de développer un véritable tourisme culturel au sein du réseau des musées.

\section{Un sujet politiquement délicat}

Aborder la question des visiteurs et des touristes dans les musées constitue un sujet délicat car il oblige à traiter de la crise que suscitent ces changements dans la culture des musées. Si ces transformations sont souhaitées par les gouvernements et que les pressions internationales sont de plus en plus fortes, les conservateurs et les professionnels qui œuvrent dans les musées opposent une résistance à ce qu'ils qualifient de «marchandisation des musées". Les transformations qui touchent l'univers des musées font d'ailleurs l'objet de débats au sein des comités nationaux du Conseil international des musées (ICOM) et d'ICOFOM international (comité international pour la muséologie). Les blockbusters et la fréquentation des institutions muséales sont donc devenus des sujets délicats, pour ne pas dire «politiquement sensibles».
Sans nous étendre sur le sujet, il convient de rappeler ici le cas ultime de cette nouvelle logique de marchandisation des musées qui défraie les manchettes internationales depuis deux ans. En 2005, le gouvernement français a choisi de confier une partie de la collection du Louvre à un nouveau musée qui sera construit à Abou Dhabi. Après avoir vendu le nom de la Sorbonne aux Émirats arabes unis, le gouvernement français négocie la location de collections et la vente de la marque de commerce "Louvre». Cette position est défendue par le gouvernement français dans un rapport de la Commission sur l'économie de l'immatériel (Lévy et Jouyet, 2006). Les conservateurs du Louvre et le corps des conservateurs de France se sont opposés à cette délocalisation des collections, invoquant plusieurs arguments, dont le fait que ces collections soient la propriété des citoyens français et le conflit d'intérêt où se place le gouvernement. Les pressions politiques ont été si fortes que les conservateurs n'ont eu d'autre choix que de se rétracter sous peine de perdre leur poste. Jean Clair, dans son dernier ouvrage, Malaises dans les musées (2007), montre bien que, au-delà du débat sur la légitimité du gouvernement de monnayer la collection du Louvre pour une somme évaluée à près de un milliard d'Euros, on se heurte à un cas de censure où les conservateurs perdent le droit de protéger les collections pour l'ensemble des citoyens. Qui peut prétendre maintenant que le mouvement de marchandisation des musées n'a pas d'effets sur le développement des musées? Une chose est certaine, nous sommes en face de transformations profondes de l'univers des musées à l'échelle internationale.

Pour trouver de nouvelles sources de financement, les musées doivent développer de nouveaux publics. Ce phénomène qui s'est généralisé dans les pays industrialisés se situe dans ce que François Mairesse (2005) identifie, dans son livre intitulé Le droit d'entrer au musée, comme la logique du discours économique. Au cours des années 1990, les musées nord-américains se sont inscrits dans le réseau des industries culturelles au même titre que le cinéma, la télévision et les arts de la scène.
Bien sûr, on peut prétendre que ce cas ne concerne que les grands pays, comme la France, la Grande-Bretagne, les États-Unis, I'Italie, la Grèce ou l'Allemagne, qui disposent de collections dites encyclopédiques couvrant l'ensemble de l'histoire de l'art. Cependant, les observateurs de I'ICOM relatent régulièrement des cas qui donnent à penser que les musées sont sur la voie du libéralisme culturel et économique. La question qui nous intéresse plus particulièrement est donc de comprendre, au-delà des discours dominants sur la fréquentation des musées, ce que nous savons concrètement des personnes qui fréquentent les institutions muséales au Québec.

\section{Connaître les publics des musées}

Ce n'est pas un hasard si l'on voit apparaître au cours des années 1990 les premières études de fréquentation et d'évaluation des visiteurs (Le Marec, 2007). Ces recherches mettent en lumière la complexité des types de publics qui fréquentent les musées. II est par ailleurs essentiel de préciser que ces études n'ont été menées que dans certaines institutions muséales. Au Québec, par exemple, on ne compte que deux musées qui disposent d'une équipe permanente dont le mandat est d'analyser les comportements des visiteurs. II s'agit essentiellement du Musée de la civilisation à Québec et du Musée canadien des civilisations à Gatineau. Par ailleurs, Parcs Canada, qui a la responsabilité d'un vaste réseau de centres d'interprétation, a également mis en place une équipe de professionnels qui mesure l'appréciation des visiteurs et les comportements des différents publics. On trouve bien sûr certains musées qui font appel à des firmes de consultants pour réaliser des études, mais celles-ci demeurent ponctuelles. De plus, cette approche ne donne pas toujours les résultats escomptés, dans la mesure où ces études sont rarement réalisées selon les mêmes paramètres, de sorte que l'analyse des résultats sur une longue période pose des problèmes méthodologiques d'interprétation des données. Ainsi, la méta-analyse (Gauthie et Pouchot, 2004) des différentes études produites au Musée d'art contemporain de Montréal de 1964 à 2003 montre bien les limites de cette approche. L'examen de l'ensemble des études 
Illustration 1

\section{Répartition des entrées selon le type d'institutions, Québec, 2005}

11871603 entrées dans les institutions muséales en 2005

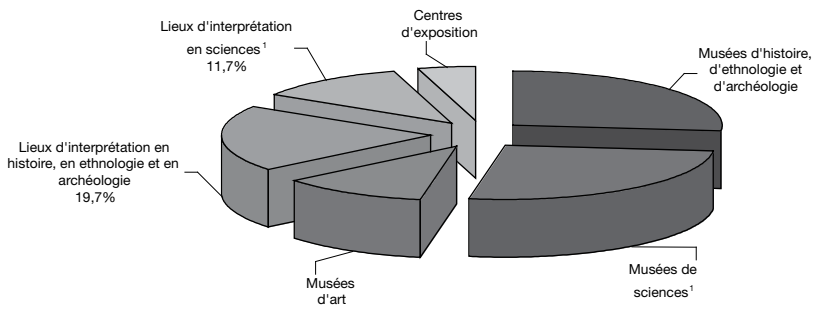

1. Sciences naturelles et environnementales, et sciences et technologie.

Source : Institut de la statistique du Québec, Observatoire de la culture et des communications du Québec, Enquête sur la fréquentation des institutions muséales.

\section{Illustration 2}

Répartition des entrées dans les institutions muséales au cours de l'année, Québec, 2005

11871603 entrées dans les institutions muséales en 2005

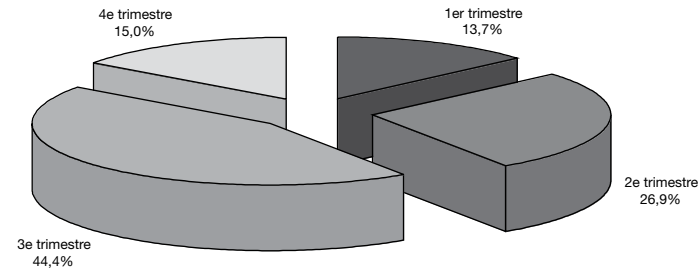

Source : Institut de la statistique du Québec, Observatoire de la culture

et des communications du Québec, Enquête sur la fréquentation des institutions muséales. réalisées au Musée d'art contemporain au cours des trente dernières années ne permet pas de cerner le profil des visiteurs. $\mathrm{Au}$ contraire, la méta-analyse réalisée par André Allaire (1999) au Musée de la civilisation montre bien que la mise en place d'enquêtes régulières, rigoureuses et comparables, permet d'observer les tendances et les transformations profondes dans les comportements des visiteurs. De manière générale, les musées québécois ne font pas d'étude continue de leurs visiteurs. Tout au plus, on constate qu'ils tiennent compte du nombre de visiteurs. Au mieux, ils réalisent des études ponctuelles pour répondre aux exigences des organismes qui financent la réalisation d'expositions ou les campagnes de promotion touristiques. Dans ces cas particuliers, les musées font appel à des ressources extérieures pour évaluer leurs publics. C'est pourquoi les musées n'arrivent pas vraiment à développer une expertise à l'intérieur même de leurs équipes. C'est d'ailleurs ce qui explique que les musées arrivent mal à poser un diagnostic critique sur les méthodes et les résultats obtenus lors de ces enquêtes.

\section{La fréquentation des musées québécois}

Il est essentiel de rappeler que nous disposons depuis peu de données précises sur la fréquentation de l'ensemble du réseau des musées québécois. L’Observatoire de la culture et des communications du Québec a en effet livré les premiers résultats sur la fréquentation des institutions muséales en 2004 (Thibault, 2004). Pour la première fois, nous disposons d'un portrait réel du contexte particulier des musées. Un examen attentif fait cependant ressortir que ce sont essentiellement des renseignements sur les flux de visiteurs qui y sont révélés. Nous avons choisi de retenir les chiffres de l'année 2005, car c'est au cours de la saison estivale 2005 que l'OCCQ a mené une première enquête sur la provenance des visiteurs qui permet de dégager un portrait des touristes dans le réseau des institutions muséales.

On constate notamment que le réseau québécois regroupait en 2005 près de 423 institutions muséales, dont 124 musées, 238 lieux d'interprétation et 61 centres d'exposition. Au cours de cette année, on a dénombré plus de 11,9 millions de visiteurs dans l'ensemble du réseau. C'est donc dire le poids relatif que représentent les musées dans les pratiques culturelles et l'industrie touristique. Cette enquête annuelle permet également de connaître les proportions de clientèle générale et de clientèle scolaire. Ces données ont d'ailleurs permis de relativiser le mythe voulant que la clientèle scolaire représente une part considérable des visiteurs des musées. Depuis que les données sont recueillies sur l'ensemble du réseau, la clientèle scolaire représente plus ou moins $10 \%$ de l'ensemble des visiteurs.
Ces données statistiques indiquent également la position relative des musées d'histoire, des musées de sciences et des musées d'art. Les résultats permettent de relativiser l'intérêt que portent les visiteurs à certains types d'expositions. On constate par exemple que les institutions d'histoire, d'ethnologie et d'archéologie étaient les plus fréquentées en 2005. Elles représentaient $46 \%$ de l'ensemble des entrées au Québec. Elles étaient suivies des musées et des centres d'interprétation en sciences qui totalisaient 4625695 entrées et représentaient $37 \%$ de la fréquentation des musées. Quant aux musées d'art et aux centres d'exposition, ils accueillaient 1,9 million de visiteurs et représentaient globalement $17 \%$ de la fréquentation du réseau.

Les données recueillies par l'OCCQ permettent également de dresser un portrait de la fréquentation par région. On y voit bien sûr se dessiner le poids relatif de Montréal et de Québec où l'on trouve le plus grand nombre de musées. La région de l'Outaouais se distingue également dans la mesure où elle abrite le Musée canadien des civilisations, qui demeure un des musées les plus fréquentés au Canada.

\section{Au-delà de la fréquentation : les pratiques culturelles des Québécois}

Enfin, l'enquête menée par l'OCCQ a permis de confirmer que la fréquentation des 
Tableau 1

Répartition des visiteurs de la clientèle générale dans les institutions muséales répondantes selon leur lieu de résidence principal et selon le type d'institutions, Québec, $3^{\mathrm{e}}$ trimestre 2005

\begin{tabular}{|c|c|c|c|c|}
\hline \multirow[t]{2}{*}{$\begin{array}{l}\text { Lieu de résidence principal } \\
\text { des visiteurs }\end{array}$} & Musées & $\begin{array}{c}\text { Lieux } \\
\text { d'interprétation }\end{array}$ & $\begin{array}{c}\text { Centres } \\
\text { d'exposition }\end{array}$ & $\begin{array}{l}\text { Toutes les institu- } \\
\text { tions muséales } \\
\text { répondantes }\end{array}$ \\
\hline & \multicolumn{4}{|c|}{$\%$} \\
\hline Québec ${ }^{1}$ & 48,0 & 73,3 & 89,7 & 56,6 \\
\hline 40 kilomètres et moins & 17,8 & 12,4 & 65,2 & 16,7 \\
\hline Plus de 40 kilomètres & 30,2 & 50,5 & 24,4 & 36,6 \\
\hline Canada (hors Québec) ${ }^{1}$ & 25,1 & 7,8 & 4,8 & 19,3 \\
\hline Ontario & 18,6 & 4,6 & 2,3 & 13,9 \\
\hline Autres provinces canadiennes & 6,3 & 3,0 & 2,5 & 5,2 \\
\hline États-Unis ${ }^{1}$ & 6,6 & 5,2 & 1,5 & 6,1 \\
\hline \multicolumn{5}{|l|}{ Nouvelle-Angleterre } \\
\hline et Atlantique Centre & 1,6 & 1,9 & 0,7 & 1,7 \\
\hline Autres états américains & 0,7 & 2,8 & 0,8 & 1,3 \\
\hline Europe $^{1}$ & 15,8 & 11,8 & 2,9 & 14,3 \\
\hline France & 7,5 & 8,3 & 1,8 & 7,7 \\
\hline Autres pays d'Europe & 1,7 & 2,5 & 1,1 & 2,0 \\
\hline Autres pays & 4,5 & 1,9 & 1,1 & 3,7 \\
\hline Total & 100 & 100 & 100 & 100 \\
\hline
\end{tabular}

1. Lorsque le total d'un type de clientèle ne correspond pas à la somme de ses composantes, cela signifie que des visiteurs ont été déclarés au total, par les institutions répondantes, sans être répartis dans les composantes.

Source : Institut de la statistique du Québec, Observatoire de la culture et des communications du Québec, Enquête sur la fréquentation des institutions muséales, «voter sur le lieu de résidence des visiteurs», Québec, 2005.

musées se concentre entre les mois de mai et août, puisqu'on y dénombre alors $57 \%$ du total des visiteurs de l'année. On constate d'ailleurs que de nombreux musées ouvrent leurs portes pour la saison estivale et ferment au début de l'automne. Ainsi, on compte 410 institutions muséales ouvertes au public au cours des mois de juillet et août, alors qu'il y en a à peine 211 au mois de janvier. Bref, ces données sont précieuses pour mesurer les fluctuations d'année en année.

Cependant, au-delà de la mesure de la fréquentation, on sait peu de choses sur le grand public qui fréquente les musées. Pour obtenir un aperçu de ces visiteurs, il faut s'en remettre aux grandes enquêtes sur les pratiques culturelles des Québécois menées par le ministère de la Culture et des Communications du
Québec depuis la fin des années 1970. Rosaire Garon, qui a dirigé ces enquêtes pendant une vingtaine d'années, a produit, avec Lise Santerre, une synthèse remarquable en 2004 en analysant les changements de comportements liés aux pratiques culturelles des Québécois de 1979 à 1999. Cette étude fait ressortir que le musée fait de moins en moins peur et qu'il tend à séduire un public qui s'est particulièrement élargi dans les grandes agglomérations au cours des deux dernières décennies. L'apparition de nouveaux grands musées a eu un effet positif sur la fréquentation. Par exemple, l'effet positif du Musée de la civilisation en 1988 y est souligné, ainsi que le fait que la scolarité et le statut socioprofessionnel demeurent les éléments clés qui déterminent la visite au musée. D'ailleurs, toutes les études démontrent que la fréquentation croît avec le niveau de scolarité (Garon et Santerre, 2004). Enfin, il semble la fréquentation de musées soit également liée à des traditions culturelles. Rosaire Garon et Lise Santerre attirent l'attention sur le fait que, de 1979 à 1999, les francophones semblent moins attirés par les musées que les anglophones et les allophones. Les études sur les pratiques culturelles démontrent par ailleurs que la fréquentation des musées tient largement aux conditions socioéconomiques et culturelles. Au-delà de ces données générales, force est de conclure que l'on dispose de peu d'information sur le profil et la provenance des visiteurs qui fréquentent le réseau des institutions muséales.

\section{Un premier portrait des visiteurs et des touristes dans les musées au Québec}

Au cours de la saison estivale 2005, l'Observatoire de la culture et des communications a mené une enquête spéciale pour connaître la provenance géographique des visiteurs (Bergeron et Thibault, 2008) de manière à mieux comprendre la place du tourisme dans la fréquentation des musées. Cette enquête a retenu un échantillon de 197 institutions muséales réparties sur l'ensemble du territoire québécois. On a d'ailleurs pris soin de distinguer les visiteurs provenant du Québec selon deux catégories, c'est-à-dire la clientèle locale, habitant à moins de 40 kilomètres de l'institution muséale visitée, et la clientèle québécoise, régionale ou provinciale, considérée comme la clientèle touristique. Quant aux autres clientèles touristiques, elles se composent des visiteurs provenant du Canada, des États-Unis, de l'Europe et des autres pays.

Pendant le troisième trimestre de 2005, les institutions muséales québécoises ont reçu plus de 5 millions de visiteurs faisant partie de la clientèle dite "générale». On constate que cette dernière serait composée à $17 \%$ de visiteurs locaux et à $83 \%$ de touristes. Selon ce projet pilote, les Québécois forment le groupe le plus important des visiteurs puisqu'ils représentent $57 \%$ de la clientèle totale. Les Québécois sont suivis des Canadiens (19\%), des Européens (14\%) et des Américains (6\%) ${ }^{2}$. 
À l'examen plus particulier des musées par catégorie on observe des profils de clientèles touristiques différents. Ainsi, les cinq grands musées, c'est-à-dire le Musée de la civilisation (MCQ), le Musée national des beaux-arts du Québec (MNBA), le Musée des beaux-arts de Montréal (MBAM), le Musée d'art contemporain de Montréal (MACM) et le Musée canadien des civilisations (MCC) ${ }^{3}$, font partie de la catégorie des musées qui accueillent le plus grand nombre de visiteurs. À eux seuls, ces cinq musées rejoignent près de la moitié des visiteurs du réseau des musées au Québec. En analysant ces résultats, on constate que les Québécois forment près de la moitié de ces visiteurs. Ils sont suivis des Canadiens, dont près des trois quarts viennent de l'Ontario. De plus, l'enquête a révélé que près de la moitié des Européens qui fréquentent les musées québécois sont d'origine française.

En somme, les visiteurs des grands musées comptent $15 \%$ de clientèle locale et $85 \%$ de touristes. II ne fait aucun doute que ces musées jouent un rôle majeur dans le réseau des institutions muséales du Québec en attirant l'essentiel des touristes provenant de l'extérieur du Québec.

\section{Une autre réalité pour les petits musées}

La catégorie des petits musées qui accueillent annuellement moins de 10000 visiteurs regroupe 227 musées, lieux d'interprétation et centres d'exposition. II est important de souligner que près de la moitié de ces institutions muséales ne sont ouvertes au public que durant la période estivale. C'est donc dire que ce groupe s'adresse principalement à une clientèle touristique. Contrairement à ce qu'on a observé pour les grands musées, les résultats de l'enquête démontrent que ces institutions sont principalement fréquentées par des touristes Québécois qui représentent huit visiteurs sur dix. Les Européens se situent au deuxième rang (11\%), devant les Canadiens (4\%) et les Américains (2\%). Encore une fois, les Français représentent une part importante des visiteurs (9\%), puisqu'ils sont plus nombreux que les Canadiens et les Américains réunis. Les visiteurs provenant

Tableau 2

\section{Répartition des visiteurs de la clientèle générale dans les institutions muséales répondantes selon leur lieu de résidence principal et selon la taille des institutions, Québec, $3^{e}$ trimestre 2005}

\begin{tabular}{lcccc}
\hline $\begin{array}{l}\text { Lieu de résidence principal } \\
\text { des visiteurs }\end{array}$ & Taille $1^{1}$ & Taille $2^{2}$ & Taille $3^{3}$ & $\begin{array}{c}\text { Toutes les institu- } \\
\text { tions muséales } \\
\text { répondantes }\end{array}$ \\
\cline { 2 - 5 } & 48,9 & 75,2 & 83,1 & 56,6 \\
\hline Québec & & & \\
40 kilomètres et moins & 15,0 & 16,6 & 33,4 & 16,7 \\
Plus de 40 kilomètres & 33,9 & 41,4 & 49,6 & 36,6 \\
\hline Canada (hors Québec) & 24,0 & 7,8 & 3,8 & 19,3 \\
Ontario & 17,6 & 4,9 & 2,4 & 13,9 \\
Autres provinces canadiennes & 6,3 & 2,7 & 1,4 & 5,2 \\
États-Unis & 6,9 & 4,7 & 1,6 & 6,1 \\
Nouvelle-Angleterre & & & & 1,7 \\
et Atlantique Centre & 1,6 & 2,3 & 0,9 & 1,3 \\
Autres états américains & 1,4 & 1,4 & 0,7 & 14,3 \\
Europe & 15,6 & 11,0 & 10,9 & 7,7 \\
France & 7,9 & 6,5 & 8,8 & 2,0 \\
Autres pays d'Europe & 1,7 & 2,8 & 2,1 & 3,7 \\
\hline Autres pays & 4,6 & 1,3 & 0,5 & $\mathbf{1 0 0}$ \\
\hline Total & $\mathbf{1 0 0}$ & $\mathbf{1 0 0}$ & $\mathbf{1 0 0}$ & \\
\hline 1. & & & & \\
\hline
\end{tabular}

1. Taille $1: 50000$ visiteurs et plus déclarés au cours de l'année 2004.

2. Taille $2: 10000$ à 49999 visiteurs déclarés au cours de l'année 2004

3. Taille 3 : moins de 10000 visiteurs déclarés au cours de l'année 2004

4. Lorsque le total d'un type de clientèle ne correspond pas à la somme de ses composantes, cela signifie que des visiteurs ont été déclarés au total, par les institutions répondantes, sans être répartis dans les composantes.

Source : Institut de la statistique du Québec, Observatoire de la culture et des communications du Québec, Enquête sur la fréquentation des institutions muséales, Volet sur le lieu de résidence des visiteurs, Québec, 2005.

d'autres pays ne représentent que 0,5\% des visiteurs.

L'enquête démontre que les touristes qui proviennent de l'extérieur du Québec visitent surtout les grands musées. Quant aux touristes européens, ils concentrent leurs visites dans les musées et les lieux d'interprétation qui traitent de l'histoire. Les Français, quant à eux, effectuent surtout des visites dans les musées et dans les lieux d'interprétation. Pour leur part, les Américains, tout comme les Français, se concentrent également dans les musées et dans les lieux d'interprétation qui traitent de l'histoire et de l'identité québécoise.

On constate que les institutions muséales situées en dehors des grands centres peuvent compter sur des clientèles locale et régionale importantes. Les données recueillies dans plusieurs régions du Québec montrent que les Québécois forment souvent le noyau fort de la clientèle touristique. Ces résultats laissent entendre que les musées seraient perçus comme des espaces identitaires pour les Québécois.

Pour l'instant, les résultats de l'enquête menée par l'OCCQ ne permettent pas de réaliser une analyse par région touristique, car les données par région demeurent inégales. Les grands musées de la région de Montréal, qui accueillent statistiquement le plus grand nombre de visiteurs, ont peu répondu à l'enquête, de sorte qu'on peut difficilement analyser le profil des visiteurs en fonction des régions touristiques. L'enquête qui doit être reprise par l'OCCQ devrait permettre de dresser un portrait plus juste des visiteurs sur l'ensemble du territoire québécois. 


\section{Illustration 3}

Répartition des visiteurs de la cientèle générale des établissements répondants ayant plus de 50000 visiteurs par année selon le lieu de résidence principal des visiteurs, Québec, $3^{\text {e }}$ trimestre 2005

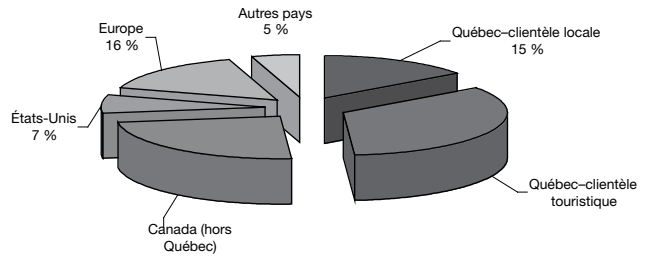

Source : Institut de la statistique du Québec, Observatoire de la culture et des communications du Québec, Enquête sur la fréquentation des institutions muséales, «voter sur le lieu de résidence des visiteurs», Québec, 2005.

\section{Illustration 4}

Répartition des visiteurs de la clientèle générale des établissements répondants ayant moins de 10000 visiteurs par année selon le lieu de résidence principal des visiteurs, Québec, $3^{e}$ trimestre 2005

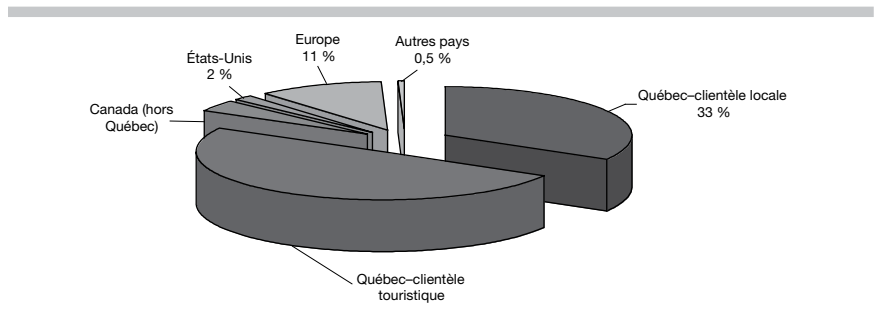

Source : Institut de la statistique du Québec, Observatoire de la culture et des communications du Québec, Enquête sur la fréquentation des institutions muséales, "voter sur le lieu de résidence des visiteurs", Québec, 2005.

\section{Au-delà du paradoxe}

Si les musées nord-américains et québécois sont reconnus pour répondre aux besoins et aux attentes des visiteurs, force est de constater qu'en réalité on en connaît très peu sur ces publics qui fréquentent les musées québécois. Une chose est certaine, aucune analyse globale ne permet pour l'instant de qualifier le profil socioéconomique et culturel de ces visiteurs. À première vue, on pourrait donc penser que nous sommes face à un paradoxe. Pourtant, les musées canadiens et québécois ont développé une culture où les processus de médiation tiennent compte de publics que l'on ne connaît pas vraiment. En fait, les musées québécois produisent des expositions ainsi que des programmes éducatifs et culturels qui tiennent compte d'objectifs de communication. Cette philosophie se traduit dans toutes les stratégies de médiation et notamment par l'accueil des visiteurs. Inspirés par les principes de Freeman Tilden (1957), les musées québécois continuent de développer des programmes éducatifs et culturels qui prolongent l'expérience de visite des expositions au-delà de la simple contemplation.

Reste maintenant à franchir une nouvelle étape pour véritablement connaître les visiteurs des musées et le profil des touristes qui fréquentent les musées québécois. Espérons que les musées répondront adéquatement aux questions de l'Observatoire de la culture et des communications du Québec lors de la prochaine enquête nationale.
Yves Bergeron est professeur en muséologie et patrimoine à l'Université du Québec à Montréal.

Suzanne Dumas est économiste spécialisée en économétrie au ministère de la Culture, des Communications et de la Condition féminine.

\section{Notes}

1 Le texte de cette conférence a été retranscrit à partir des bandes enregistrées et publiées par Mairesse et al. (2008).

2 Les autres pays ne sont pas représentés de manière significative.

3 II faut comprendre que les moyens dont disposent ces cinq grands musées sont beaucoup plus importants que ceux des autres musées composant le réseau. Trois de ces institutions muséales sont régies par la Loi sur les musées nationaux. Comme elles sont mandataires de l'État, ces institutions ont des responsabilités nationales dont la loi précise les fonctions et les pouvoirs. La mission propre à chaque musée constitue en quelque sorte l'engagement social que prend chaque institution face à l'État et aux citoyens.

\section{Bibliographie}

Allaire, André (1999), Portrait statistique de la fréquentation et des visiteurs du Musée de la civilisation 1988-1999, Québec, Musée de la civilisation, Les Cahiers de recherche du Musée de la civilisation, Recherche et évaluation.

Arpin, Roland (1992), Le Musée de la civilisation. Concept et pratiques, Québec, MultiMondes.

Arpin, Roland (1997), Des musées pour aujourd'hui, Québec, Musée de la civilisation.
Bergeron, Yves et Marie-Thérèse Thibault (2008), "Publics et Touristes dans le réseau des institutions muséales au Québec ", Statistiques en bref, $n^{\circ} 36$.

Clair, Jean (2007), Malaise dans les musées, Paris, Flammarion, coll. "Café Voltaire».

Garon, Rosaire et Lise Santerre (2004), Déchiffrer la culture au Québec. 20 ans de pratiques culturelles, Québec, Les Publications du Québec.

Gauthier, Michelle et Stéphanie Pouchot (2004), Rapport d'étude. Une relecture des rapports d'études de clientèles menées au Musée d'art contemporain de Montréal depuis sa création, Montréal, DigiCulture.

Le Marec, Joëlle (2007), Publics et musées. La confiance éprouvée, Paris, L'Harmattan.

Lévy, Maurice et Jean-Pierre Jouyet (2006), L'économie de l'immatériel. La croissance de demain, Paris, La Documentation française.

Mairesse, François (2005), Le droit d'entrer au musée, Bruxelles, Éditions LABOR.

Mairesse François, Bernard Deloche, Marshall McLuhan, Harley Parker et Jacques Barzun (2008), Le musée non linéaire. Exploration des méthodes, moyens et valeurs de la communication avec le public des musées, Lyon, Aleas.

MCCQ (Ministère de la Culture et des Communications du Québec) (2000), La politique muséale. Vivre autrement la ligne du temps, Québec, MCCQ.

OCCQ (Observatoire de la culture et des communications du Québec) (2007), "État des lieux du patrimoine, des institutions muséales et des archives", Les institutions muséales du Québec, redécouverte d'une réalité complexe, Cahier 3, p. 24.

Thibault, Marie-Thérèse (2004), «La fréquentation des institutions muséales au Québec en 2003 ", Statistiques en bref, n० 6, mai.

Tilden, Freeman (1957), Interpreting our Heritage, Chapel Hill, Carolina Press. 